\title{
AUSGEWÄHLTE KARRIEREN DER BÖHMISCHEN JOHANNITER IM 14. JAHRHUNDERT
}

\author{
KEYWORDS
}

Hospitallers; Czech priory; Prague convent; nobility as members of the Order

目 ie Karrieren der Johanniter von Böhmen und Mähren am Ende des 12. und während des 13. Jahrhunderts sind - mit der einzigen Ausnahme eines der Begründer des Ordens, des Propstes Martin - kaum zu verfolgen, denn dazu fehlt es an aussagekräftigen Quellen. ${ }^{1}$ Erst an der Wende zum 14. Jahrhundert wird dies im Falle des böhmischen Priors Hermann von Hohenlohe möglich, der aber nicht böhmischer Abstammung war. Er entstammte der linken Ehe eines Adligen aus dem Umfeld Rudolfs von Habsburg. ${ }^{2}$ Erst im 14. Jahrhundert werden manche Personen häufiger in den Quellen genannt und ihre Karrieren im Orden können rekonstruiert werden. Man darf auch den Umstand nicht unterschätzen, dass der böhmisch-mährische Zweig des Johanniterordens in nationaler Hinsicht eine interessante Entwicklung durchlief. Während am Anfang die Mitglieder böhmischer Abstammung dominierten, setzten sich vor allem in den städtischen Kommenden seit der Mitte des 13. Jahrhunderts als Konsequenz der Siedlungsbewegungen deutschsprachige Mitglieder durch. In Prag stellten sie z. B. im zweiten Dezennium des 14. Jahrhunderts klar die Mehrheit. ${ }^{3}$ Auch dieser Stand der Dinge

1 Vgl. M. Skopal, Založení komendy johanitư na Malé straně. Príspèvek $k$ otázce príchodu rádu do Cech, Pražský sborník historický 26 (1993), S. 7-36; L. Jan, “... mortuus est persecutor noster Saladinus”. (K zpi̊sobu komunikace mezi českými zemémi a Palestinou ve 12. a 13. století), Sborník prací filozofické fakulty brněnské univerzity / Studia minora Facultatis Philosophicae Universitatis Brunensis C 44 (1997), S. 17-35; Z. Hunyadi, The Hospitallers in the Medieval Kingdom of Hungary c. 1150-1387, Budapest 2010, hier S. 69-70.

2 Zu Hermann von Hohenlohe zuletzt L. Jan, Hermann z Hohenlohe, rádce a vyslanec českého krále Václava II., Sborník prací filosofické fakulty brněnské univerzity / / Studia minora Facultatis Philosophicae Universitatis Brunensis C 43 (1996), S. 17-35.

3 Vgl. L. Jan, Die Johanniter in Böhmen: Bild des Lebens, in: Vergangenheit und Gegenwart der Ritterorden. Die Rezeption der Idee und die Wirklichkeit, hrsg. v. R. Czaja, J. Sarnowsky (Ordines militares. Colloquia Torunensia Historica XI), Toruń 2001, S. 186-187. 
sollte sich aber ändern. Es ist auch wichtig daran zu erinnern, dass es an der Wende der 20er und 30er Jahre des 14. Jahrhunderts gelang, die dauerhafte Besetzung der Würde des böhmischen Priors mit einem heimischen Kandidaten zu sichern, obwohl in dieser Sache mehrfach ein Streit mit der Führung des Ordens ausbrach. ${ }^{4}$ Als „heimisch“ galten im Folgenden nicht nur die Kandidaten aus Böhmen und Mähren, sondern auch aus Schlesien, was mit dem Prozess des allmählichen Anschlusses der schlesischen Fürstentümer an die Länder der böhmischen Krone zusammenhing.

Ende Januar 1371 starb unweit von Prag, in der Festung von Uhř́něves/Aurzimowes, Johann von Zweretitz (tsch. Zviŕetice), der Prior des Ritterlichen Ordens Sankt Johannis vom Spital zu Jerusalem in Böhmen, Mähren, Polen und Österreich. Wir wissen nicht, in welchem Alter er starb, und bis auf einige Informationen sind wir nicht im Stande sein Leben im Detail zu rekonstruieren. Die ganz wenigen Splitter sagen uns, dass er einem Zweig des weit verbreiteten Adelsgeschlechts der Markwartinger angehörte, das relativ einzigartig ein doppeltes Wappenzeichen führte, den geteilten Schild und einen Löwen. Er war Komtur in Manětín und in Prag, und in den Jahren 1367-1371, also eine relativ kurze Zeit, bekleidete er die Würde des böhmischen Priors, in der er seinen Verwandten aus einem anderen Zweig des Geschlechts Gallus (tsch. Havel) von Löwenberg (tsch. Lemberk, Lvová) ablöste. Am Sterbebett von Johann weilten außer einigen Ordensgeistlichen und Dienern drei reife Männer. Neben dem Prager Komtur Pesslin waren dies der Zittauer Komtur Henslin von Prag und der Komtur von Jungbunzlau-St. Veit Paul Kabát. ${ }^{5}$ Wir konzentrieren uns auf den Erstgenannten.

Peter wird erstmals am 25. Mai 1363 als Prager Komtur genannt, wo er gemeinsam mit dem Konvent bei der Marienkirche bezeugt, dass ihnen der Johanniter Johann von Strakonitz ein Schock des Zinses aus dem Ackerboden (haereditas) im Dorf Pačejov vermacht hatte. ${ }^{6}$ Es ist unbestreitbar, dass er im Amt Johann von Zweretitz ablöste, der zuletzt als Komtur am 21. Januar 1362 genannt wird.7 Zwischen diesem Datum und dem 25. Mai 1363 wurde Peter also zum Komtur bei

4 Dazu vgl. K. Borchardt, The Hospitallers, Bohemia, and the Empire, 1250-1330, in: Mendicants, Military Orders and Regionalism in Medieval Europe, ed. J. Sarnowsky, Aldershot 1999, S. 201-231, hier 221-228; Jan, Die Johanniter in Böhmen (wie Anm. 3), S. 189-190; J. Mitáček, Die böhmische Provinz des Johanniterordens und die Luxemburgem 1310-1437, in: Die geistlichen Ritterorden in Mitteleuropa. Mittelalter, hrsg. v. K. Borchardt, L. Jan (Edice Země a kultura ve střední Evropě 20), Brno 2011, S. 99-113.

5 Nationalarchiv Prag, Archiv des Malteser Grosspriorats (weiterhin: NA Praha $\check{R} M$ ), Urkunden, Nr. 2233, 2235, 2820, 2836, 2516.

6 NA Praha ŘM, Urkunden, Nr. 2212.

7 Liber primus Confirmationum ad beneficia ecclesiastica Pragensem per archidioecesim ... ab anno 1354 usque 1362, op. F. A. Tingl, Pragae 1867, S. 170. 
der Marienkirche in Prag ernannt, während sein Vorgänger Johann von Zweretitz wieder als Komtur nach Manětín ging, wo er schon einmal, zu Anfang der 50er Jahre diese Funktion ausgeübt hatte. ${ }^{8}$ Alle diese Verschiebungen vollzogen sich natürlich in Übereinstimmung mit dem Willen des Landespriors Gallus von Löwenberg; Peter war zu dieser Zeit gerade vierzig Jahre alt, wenn wir seiner Angabe von 1373, er habe das Alter von 50 Jahren erreicht, Glauben schenken, die er während der Visitation der Johanniter-Häuser in der Prager Erzdiözese 1373 machte. ${ }^{9}$

Pesslin wurde als Komtur sogar in der Ordenszentrale bestätigt. Denn in einer sonderlichen Urkunde, die auf Rhodos am 1. Oktober 1364 nach einem Ordensformular niedergeschrieben wurde, übergab der Großmeister Peter die Verwaltung des Prager Hauses (preceptoria seu baiulia) für zehn Jahre mit der Verpflichtung der jährlichen Bezahlung von Responsionen und weiteren Abgaben zum Termin des Provinzkapitels. ${ }^{10}$ In den nächsten Jahren begegnen wir Peter als Repräsentanten des Prager Hauses bei mannigfaltigen Handelstransaktionen und Verfügungen von Personen aus städtischem Milieu zugunsten der Johanniter. Peter hängte den Urkunden gewöhnlich sein Siegel an, es überrascht daher ein wenig - trotz seiner Zugehörigkeit zu einem geistlich-ritterlichen Orden -, dass sein Siegelbild rein religiöser Natur ist. Darüber hinaus geht es nicht um ein geläufiges Motiv aus dem thematischen Umfeld um Johannes den Täufer, sondern um ein christologisches Bild. Denn im Siegelfeld ist das Veraikon abgebildet, das Schweißtuch, auf dem sich das Gesicht Jesu eingeprägt hat. In der Zeit, von der wir sprechen, wurde eines der Tücher in der römischen Petersbasilika aufbewahrt; von hier aus wurden Kopien verbreitet, und das Motiv verbreitete sich auch in Gestalt von zahlreichen Tafelbildern mit der kennzeichnenden Darstellung Christi mit langen herabfließenden Haaren und Gabelbart. In der Zeit, wo Peter sein Siegelbild wählte, war aber bei St. Veit diese einigermaßen abweichende Darstellung Christi (ohne Tuch) noch nicht bekannt. Peter genannt Pesslin musste daher dem Veraikon, also dem „wahren Bild“, anderswo begegnet sein und diese Begegnung musste bei ihm zweifellos einen tiefen, wahrscheinlich sogar mystischen Eindruck hinterlassen haben. Laut der Inschrift: s.petri.com. de praga ordis scti iohis, in der vollen Fassung also sigillum petri commendatoris de praga ordinis sancti iohannis, schaffte sich Peter ein Typar erst nach seiner Ernennung zum Prager Komtur an, und es kann nicht ausge-

8 Als Komtur in Manětín wird Johann von Zweretitz wieder am 8. März 1364 und am 26. August 1365 erwähnt, vgl. NA Praha ŘM, Urkunden, Nr. 2213, 2220.

9 V. Novotný, Inquisitio domorum hospitalis s. Johannis Hierosolimitani per Pragensem archidiocesim facta anno 1373 (Historický archiv 19), Praha 1901, S. 21.

10 NA Praha ŘM, Urkunden, Nr. 2216. 
schlossen werden, dass die relativ fortgeschrittene Ausführung keinem heimischen Gravierer zuzuschreiben ist, sondern eher einem Handwerker aus Südeuropa. ${ }^{11}$

Was verrät uns Pesslins Aussage vor den Beamten des Erzbischofs über ihn? Nicht viel, aber die Aussage des Komturs ist die zweitinhaltsreichste nach den Antworten des Priors Nikolaus. Zunächst zählt Peter alle Ordenshäuser auf dem Gebiet der Prager Diözese auf, ohne ein einziges auszulassen, er beging nur den kleinen Fehler, wenn er die Pfarrei in Pičín als abhängig von Strakonitz bezeichnete, ähnlich wie es bei der Pfarrei von Horažd'ovice der Fall war. Pičín aber hatte damals schon die Stellung eines selbstständigen Ordenshauses mit einem Komtur an der Spitze. Diesen Fehler begingen aber mehrere Zeugen aus verschiedenen Kommenden. Seinen Namen nannte er in der Form „Peter genannt Pesslin“ und er gab auch an, dass er 50 Jahre alt war. Er sollte auch über die Namen und die Zahl der Ordensgeistlichen seines Hauses aussagen. Hier machte er sich die Antwort leichter und sagte lediglich, dass hier 15 Priester, ein Diakon, ein Unterdiakon und zwei Akolyten wirken. Neun Ritterbrüder erwähnte er aber namentlich auch mit ihrem Alter.

Im „wirtschaftlichen“ Teil sind seine Antworten relativ treffend, er vergaß nicht zu betonen, dass sich sein Haus und die zugehörigen Höfe auf dem Lande „auf öffentlichen Orten und Wegen“ befanden, welcher Umstand hohe Kosten verursachte. Eine weitere Belastung seines Hauses hing mit den häufigen Besuchen des böhmischen Priors und seines großen Gefolges zusammen, die sich vor allem im Zusammenhang mit den Angelegenheiten ergaben, die der Ordenswürdenträger mit dem Kaiser und anderen Fürsten zu besprechen hatte. Das Haus „am Ende der Brücke" besuchten aber häufig auch weitere Komture, die hier unterkamen und die Ausgaben des Hauses erhöhten.

Auf die Frage, für welche Summe das Haus verpachtet werden könnte, wenn hier nicht die Ritter-Laien wären, sondern nur die Geistlichen, antwortete er, dass es nicht möglich wäre die Ertrage und Einnahmen des Hauses für viel Geld zu verpachten, wenn nicht die erwähnten Belastungen behoben werden (dazu gehörte auch die Entrichtung von Steuern und Responsionen sowie die Auszahlung von jährlichen Löhnen, von Pragern vorausbezahlt), und dass es anstatt von Ritterbrüdern, die verschiedene Ämter in der Wirtschaft verwalteten, sonst nötig wäre Laien anzuwerben. ${ }^{12}$

11 Die Siegel des Komturs Peter sind bei mehreren Urkunden erhalten, z. B.: NA Praha R̆M, Urkunden, Nr. 2212 (25. Mai 1363); Nr. 2215 (1. Mai 1364; Nr. 2226 (8. Juni 1367); Nr. 2836 (18. Januar 1371).

12 Novotný (wie Anm. 9), S. 21-23. 
Die Antworten von Peter waren zwar zutreffend, doch relativ knapp. In dieser Weise konnte ein praxisorientierter und energischer Mann antworten, der das Tun dem Sprechen vorzog. Der priesterliche Bestandteil seines Hauses stellte für ihn lediglich eine farblose Gruppe dar und „nur die Ritter hatten Namen“. Hier begegnen wir aber nicht einem Wegsehen, die sich aus seiner Abstammung ergab, eher war seine Haltung durch die Zugehörigkeit zu einer anderen Statusgruppe im Orden bestimmt. Die Abstammung konnte schon deshalb nicht der Grund sein, weil er höchstwahrscheinlich nicht aus einer Adelsfamilie stammte, die ihn für das Rittertum vorbestimmt hätte.

Wenn wir der Angabe über sein Alter Glauben schenken, d. h. 50 Jahre, wurde er im Jahre 1323 geboren. Als seinen Geburtsort gaben die Zeugen Peter Harrer, Prior Nikolaus und der Schaffer Fridlin Prag an. Er gebrauchte weder ein Prädikat noch ein Siegel mit Wappen, nach dem er identifiziert werden könnte. Gleichwohl gilt seine Abstammung aus einer Prager Bürgerfamilie als wahrscheinlich. Sein Name war Peter, er wurde aber Pesslin genannt, also mit der deutschen heimischen Form seines Taufnamens. Er entstammte daher zweifelsfrei einer deutschen Familie. Unbestritten war seine Familie exponiert, wir können sogar voraussetzen, dass die Familie gewisse Beziehungen zum Johanniterorden hatte. In den heimischen Quellen wird er vor dem Jahre 1363 - wo er zum ersten Mal mit der Würde des Komturs in Prag genannt wird - niemals als Ordensbruder erwähnt. Dafür bieten sich zwei mögliche Erklärungen an. Entweder hielt er sich lange Zeit auf Rhodos auf, oder er trat dem Orden erst kurz vor diesem Datum bei und seine vorherige erfolgreiche weltliche Laufbahn oder vielleicht die Gönnerschaft der Mächtigen der damaligen Welt prädestinierte ihn im Vorhinein für eine der führenden Stellen im Orden.

Zuletzt begegnen wir Pesslin in der Führung der Prager Kommende am 6. Oktober 1374, wo aufgrund seiner Präsentation ein weiteres Mitglied des Prager Konvents aus dem Jahr 1373 anstatt des zurückgetretenen Bruders Johann ins Amt des Kaadener Pfarrers eingeführt wurde, Nikolaus Stach. Pesslin agierte hier aber als Vertreter des Generalpriors, der über das Präsentationsrecht verfügte. Es ging nicht um die Vertretung ad hoc, vielmehr scheint es, dass Pesslin ein tatsächlicher Vertreter für Böhmen war ${ }^{13}$, denn schon am 24. Februar 1373 verhandelte er auf dem erzbischöflichen Konsistorium „im Namen seines und aller Klöster in der Prager Provinz" mit den Kollektoren der Papstzehnten, dem Dekan vom St. Apollinaris und dem erzbischöflichen Generalvikar Johann Kantor, darüber, dass er in drei Monaten nach dem genannten Tage die Zehnten in der Höhe von

13 Libri confirmationum ad beneficia ecclesiastica Pragensem per archidioecesim (weiterhin: LC), vol. III-IV, ed. J. Emler, Pragae 1879, s. 21. 
400 Gulden bezahlen sollte. ${ }^{14}$ Damals könnte seine Stellung sehr wichtig gewesen sein, denn Ziemowit von Teschen war im Amt des böhmischen Priors noch nicht bestätigt.

Nach dem 6. Oktober 1374 verfügen wir über keine weiteren Nachrichten zu Pesslin. Dabei ist zu beachten, dass am 1. Oktober 1374 die zehn Jahre zu Ende gingen, für die ihm das Prager Haus anvertraut wurde. Da die Eintragungen in die Konfirmationsbücher mit einer gewissen Verspätung erfolgten, ist es möglich, dass Pesslin schon am 6. Oktober 1374 nicht mehr das Amt des Prager Komturs bekleidete. Sein weiteres Schicksal kann man zwar nicht verfolgen, doch es ist wahrscheinlich, dass er das Amt nach dem Ablauf der ordentlichen Frist verließ. In der Mitte des Jahres 1375 bekleidete die Komturswürde bereits Hereš von Zweretitz, ein weiterer Spross des Geschlechtes der nordböhmischen Markwartinger. ${ }^{15}$

Dem einundsechzigjährigen Johann von Kladrubce, der zur Zeit der Visitation Prager Konventuale war, begegnen wir zum ersten Mal am 2. Februar 1362. An diesem Tag erlaubte der Prior des Ordens für Böhmen, Mähren, Polen und Österreich Gallus von Löwenberg während seines Aufenthaltes in der Strakonitzer Kommende dem Bruder Johann von Kladrubce auf Lebenszeit einen Hof mit einem Lahnen im Dorf Mnichov zu besitzen, den Johann mit der Zustimmung des Priors aus den finanziellen Mitteln kaufte, die er von seinem Vater geerbt hatte. Ferner erwarb er einige Ländereien und den Oberen Teich unter dem Wald Hůl. Nach dem Ableben Johanns sollte der Hof mit den Ländereien der Kommende in Strakonitz zukommen, der Teich war für die Aufbesserung der Beköstigung der hiesigen Brüder bestimmt; mit anderen Worten stellte er die Pitanz dar. Falls Johann in ein anderes Ordenshaus versetzt wird - und dieser Angabe ist klar zu entnehmen, dass er zur Zeit der Ausstellung der Urkunde Strakonitzer Konventuale war - konnte er die besagten Güter jedem Ordensbruder von Strakonitz zur Nutzung auf Lebenszeit anvertrauen. ${ }^{16}$ Der Teich nahe dem Wald genannt Hůl wird uns auf einer weiteren Reise durch die Zeit begleiten. Vier Jahre früher, am 16. März 1358, zedierte der Strakonitzer Komtur Jakob mit seinem Konvent den natürlichen Brüdern Zdimir (Sdymerius) und Johann den Ertrag von zwei Teichen nahe des Waldes genannt Hůl, die beide Brüder auf eigenen Gütern aus ihrem Eigentum angelegt hatten, das sie mit der Genehmigung des Ordens behalten durften. Nach dem Ableben eines der Brüder sollte der Strakonitzer Kommende als Pitanz der Untere Teich zukommen, nach dem Tod des anderen auch der Obere Teich. Dafür sollte für beide Brüder, wie es ansonsten üblich war, die jährliche

14 Soudni akta konsistoře pražské I (1373-1379), ed. F. Tadra, Praha 1893, Nr. 52, S. 11.

15 NA Praha $\check{R} M$, Urkunden, Nr. 2239, 2242, 2244.

16 NA Praha ŘM, Urkunden, Nr. 2432. 
Andacht gefeiert werden. ${ }^{17}$ Es ist unbestreitbar, dass in diesem Falle der zweite Bruder mit Johann von Kladrubce zu identifizieren ist.

Das Schicksal von Johann können wir weiter verfolgen. Im Jahre 1369 (entweder am 16. Januar oder am 4. September) bewilligte der Prior Johann von Zweretitz während seines Aufenthaltes in Orlovice in Mähren (anscheinend fand hier eine Tagung des Provinzkapitels statt) Johann, zu dieser Zeit Komtur in Leobschütz (pol. Glubczyce, tsch. Hlubčice), auf Lebenszeit den Teich unter dem Wald Hůl (d. h. Stock) und weitere Ländereien zu nutzen, ungeachtet der Tatsache, wohin er vom Orden abgeordnet wird. Es wird daran erinnert, dass Johann diese Güter bislang aufgrund der Bewilligung des ehemaligen Priors Gallus von Löwenberg besaß. Als Komtur von Leobschütz musste Johann von Kladrubce 1368 eine äuBerst unangenehme Angelegenheit lösen, den Streit des Johanniterordens mit der Stadt Troppau, der das hiesige Spital betraf. Es ging um den Besitz der Steinmühle unter dem Ort Kunčice und ferner um das Asylrecht der Troppauer Spitals, das anscheinend aufgrund einer vom Verwalter des Spitals und Günstling des Fürsten Markolt hergestellten Urkundenfälschung auch für Mörder, Diebe und Ausschreiter, somit eigentlich für die „professionellen“ Verbrecher bestimmt war. Von 1373, als die Visitation stattfand, bis zum Jahr 1387 vergingen fünfzehn Jahre und auf dem römischen wie auch auf dem böhmischen Thron saßen andere Herrscher. Johann von Kadrubce war zu dieser Zeit 75 Jahre alt und man würde sich sagen: eine gute Zeit sich zur Ruhe zu setzen. So war das aber nicht, denn Johann war in diesem Jahr Komtur in Manětín; es blieben ihm aber nicht mehr viele Tage übrig. Markolt von Vrutice, der Komtur von Strakonitz, bezeugte mit seinem Konvent am 6. Februar 1387 mittels einer Urkunde, dass Johann von Kladrubce, Komtur von Manětín, mit der Zustimmung des Ordenspriors, des Teschener Fürsten Ziemowit, dem Konvent von Strakonitz zur Aufbesserung den Teich Hůl unter dem Wald schenkte. Die Brüder sollten viermal jährlich, am Valentinstag, am Tag nach St. Stanislai, am Vorabend des Hl. Laurentius und am Tag nach Martini, die jährliche Andachtsmesse für die Seelen seiner Eltern und Vorfahren lesen, auch für seine Seele, nach seinem Tod, wie auch für seinen Bruder Zdimir, für den als geistliche Stiftung bereits früher der Untere Teich zediert wurde. So nahm Johann Abschied vom Oberen Teich unter dem Wald Hůl, und es kann schwerlich bezweifelt werden, dass dies im Rahmen einer Art Ausgleichs vor dem Abgang aus dieser Welt geschah. ${ }^{18}$ Johann von Kladrubce wurde irgendwann nach dem

17 NA Praha ŘM, Urkunden, Nr. 2423.

18 NA Praha R̆M, Urkunden, Nr. 2451. 
18. September 1383, wo er das letzte Mal in der Funktion genannt wird, auf dem Posten des Komturs von Manětín von Beneš von Ronov abgelöst. ${ }^{19}$

Wie bereits gesagt, hatte Johann einen Bruder Zdimir, der noch am Anfang des Jahres 1358 am Leben war, vier Jahre später war er aber höchstwahrscheinlich tot. Auch er ist nicht zu den ganz anonymen Gestalten zu zählen. Nicht lange vor dem Jahre 1349 wurde er zum Protagonisten eines kleinen Dramas, das auf seinem Leib dauernde Spuren hinterlassen hat. Den Einträgen der Papstregister vom 26. Januar 1349 ist zu entnehmen, dass zwei Laien, Johann Chunad von Billowitz (tsch. Velké Bílovice) und Paul Goblin von Brünn, bereits früher „befallen durch unbesonnene Wut" den Bruder Zdimir, den Komtur des Johanniterordens von Mutěnice, überfielen und blutig an vielen Körperteilen verletzten (Mutěnice ist aber mit dem lateinischen Wort Crux bezeichnet, also tschechisch križ und deutsch Kreuz; diesen Namen nahm das Dorf während der am ehesten von den Johannitern vollzogenen Kolonisierungsaktivitäten an). Als Folge war der Komtur an einem Glied gelähmt. ${ }^{20}$ Dieser Angriff ereignete sich bestimmt schon einige Jahre vor dem Anfang des Jahres 1349, am ehesten zwischen 1345-1347, denn Zdimir war am 28. Oktober schon soweit gesundet, dass er das Amt des Komturs einer weiteren südböhmischen Kommende ausüben konnte, nämlich in Přibice. Denn am genannten Tag gab der Landeshauptmann von Mähren Wilhelm von Landstein (tsch. Landštejn) eine Urkunde aus, in der er behauptet, dass vor ihm der Komtur von Přibice Zdimir und der Pleban des Spitals Johannes' des Täufers in Altbrünn mit dem Gesuch erschienen seien mit der Bitte, die Urkunden zu bestätigen, die der Orden vom römischen und böhmischen König Karl IV. betreffs der Freiheiten des Dorfes Přibice erlangte. ${ }^{21}$ Im Falle von beiden Wirkungsstätten von Zdimir, der Kommende in Kreuz/Mutěnice, die ursprünglich der Bestandteil des Besitzes der Kommende von Orlowitz (tsch. Orlovice) war ${ }^{22}$, wie auch der Kommende von Přibice ging es nur um ein Dorf im fruchtbaren Gebiet Südmährens, mit einer Kirche und einem Ordenshaus, das wahrscheinlich nahe der Kirche stand und sich nicht viel von den mäßig befestigten Häusern der umliegenden kleinen Adeligen unterschied. Im Falle von beiden Dörfern war es aber wichtig, dass in den Weingärten annehmbare Trauben ausreiften.

19 LC III-IV, s. 158.

20 Acta Clementis VII. pontifici Romani 1342-1352 (weiterhin: CDM), op. L. Klicman (Monumenta Vaticana res gestas Bohemicas illustrantia I), Pragae 1903, Nr. 1079, S. 598-599.

21 CDM VII, Nr. 863, S. 620. Vgl. L. Jan, Starobrněnští johanité a ves Pribice, Forum Brunense 1995/1996, S. 21.

22 Vgl. L. Jan, Ivanovice na Hané, Orlovice a johanitský rád (Príspèvek k poznáni struktury a ekonomiky rytiřrských duchovnich rádů do konce 15. století), Časopis Matice moravské 111 (1992), s. 221-222. 
Der Komtur von Strakonitz Nikolaus und der Pleban von Horazdiowitz (tsch. Horažd'ovice) Prokop bestätigten am 28. Juni 1394, dass der cliens Udalrich (Oldřich) von Kladrubce dem Ordenshaus von Horažd'ovice (es ging eigentlich um ein großes Pfarrhaus, wo sich gewöhnlich einige Brüder aufhielten und das von der Kommende von Strakonitz abhängig war) 10 Schock Groschen gab für die Andachtsmessen und Vigilien, die für die Seele des Ordensmitgliedes Johann von Kladrubce und alle Vorfahren von Udalrich gelesen werden sollten, und zwar am Geburtstag Johannes des Täufers (24. Juni) und am St. Gallus-Tag (16. Oktober) ${ }^{23}$ Es kann nicht daran gezweifelt werden, dass Johann kurz vor diesem Datum verstorben war und sein Verwandter, am ehestens sein Neffe, ihm auf diese zu dieser Zeit ganz geläufige Weise die Ehre erwies. Unzweifelhaft war er älter als 80 Jahre, wobei ihn dieses patriarchische Alter zu einer Ausnahme nicht nur im Orden, sondern auch in der ganzen Gesellschaft machte. Es lässt sich nicht übersehen, dass sich seine Lebenszeit annähernd mit der Lebenszeit einer der bedeutendsten Persönlichkeiten des Ordens im 14. Jahrhundert deckte. Juan Fernández de Heredia wurde um das Jahr 1310 geboren, den Johannitern trat er 1328 bei, er bekleidete viele bedeutende Würden auf dem Gebiet des heutigen Spaniens und Südfrankreichs, und seit 1377 bis zu seinem am 1. März 1396 erfolgten Tod in Avignon war er Großmeister des Ordens.

Johann und auch sein Bruder entstammten dem Dorf Kladrubce, das westlich von Lnáře oder westnordwestlich von Blatná liegt. Es ist nicht zu bezweifeln, dass auf diesem Gebiet die sog. vladyken, terrigenae oder andere Kleinadeligen in einem gewissen Abhängigkeitsgrad von dem mächtigsten Geschlecht der Bawor von Strakonitz lebten. Ihre Güter besaßen sie oft mit dem Willen des Strakonitzer Herren, wobei die Wehrpflicht sie zu seinen Mannen machte. Diese Klientel hielt sich oft im Strakonitzer Sitz der Bawor auf, wo sie mit den hiesigen Johannitern in Kontakt kamen, die sich aufgrund einer Entscheidung Bawors I., die er irgendwann zu Anfang der 40er Jahre des 13. Jahrhunderts traf, mit ihren weltlichen Herren ihre Burg teilten. So entstand noch vor der Mitte des 13. Jahrhunderts die Kommende von Strakonitz, deren Konventuale im Jahre 1362 Johann von Kladrubce war. Wahrscheinlich traten er und auch sein Bruder hier dem Orden bei. Sie waren nicht die einzigen Söhne von Kleinadeligen aus der Nachbarschaft, für die ihre Eltern oder das Schicksal die Karriere im Orden der ritterlichen Mönche gewählt hatten. Im Jahre 1365 wird Protiva von Štěkeň erwähnt, anscheinend ein Ordensritter, während 1373 war der bereits genannte Blasius (Blažek) von Štěkeň als Priester im Prager Konvent tätig, beide waren wahrscheinlich Sprosse einer armen Nebenlinie des Geschlechtes Bawor, zu 1374 begegnen wir dem Ordens-

23 NA Praha ǨM, Urkunden, Nr. 2141. 
mitglied Johann von Srdov. ${ }^{24}$ Diesem Milieu entstammte höchstwahrscheinlich auch der Jungbunzlauer Komtur und für eine gewisse Zeit als Vertreter des Priors in Böhmen nachweisbare Paul Kabát, dessen Verwandter Peter Kabát von Krty 1368 Lehnsmann der Strakonitzer Kommende war. ${ }^{25}$

Zum Schluss kann zusammenfassend gesagt werden, dass unter den Ritterbrüdern des Johanniterordens in der böhmischen Ballei des böhmischen Priorats am deutlichsten drei Gruppen von Personen identifiziert werden können, deren Mitglieder manche Kennzeichen gemeinsam haben. Die erste Gruppe sind die Angehörigen des verzweigten Geschlechtes der Markwartinger (Herren von Löwenberg/Lemberk, von Zweretitz/Zviŕetice, von Waldstein/Valdštejn, von Wartenberg/Vartenberk), das am Anfang einer der zwei Kommenden in Jungbunzlau stand, es geht also um eine traditionelle Geschlechtsangelegenheit, um die enge Verknüpfung eines der großen Geschlechter aus dem Hochadel mit dem Orden. ${ }^{26}$ Die zweite Gruppe stellen die Angehörigen der kleineren Nobilität aus dem Strakonitzer Gebiet, deren Familienmilieu an die Herren von Strakonitz oder direkt an die Strakonitzer Kommende gebunden war. Es geht also um die Klienten oder Mannen dieser zwei Subjekte, deren Bindung sich aus der Abhängigkeit ihrer Familien vom Gründer des Strakonitzer Hauses oder vom Haus selbst ergibt. Die dritte Gruppe sind dann die Ritter, die den wohlhabenden Prager Bürgerfamilien entstammten. Durch den Eintritt in den Johanniterkonvent bot sich für diese Männer die Möglichkeit zur Versorgung, gegebenenfalls zur Erlangung der ritterlichen Würde oder von Kriegserfahrung. Darüber hinaus kommen noch Ordensangehörige aus einigen Geschlechtern in anderen Teilen von Böhmen und Mähren vor, ohne dass wir zu einer genauen Vorstellung gelangen können, aus

24 Über Protiva (Protiwa de Schekem) spricht ein Schreiben des Kopiars der Johannitergroßmeister vom 8. November 1365; für die zur Verfügung gestellten Texte, die für eine MGH Edition vorbereitet werden, bedankt sich der Verfasser Prof. Dr. Karl Borchardt. Blažek von Štěkeň wird erwähnt in der Visitation vom Jahre 1373, siehe Novotný (wie Anm. 9), S. 24, 25, 29, und Johann von Srdov wird mit seinem gebürtigen Bruder Přibík und dem verstorbenen Vater Něpr in der Urkunde des se Komturs Johann Schreiber vom 16. Oktober 1374 erwähnt, vgl. NA Praha R̆M, č. 2443. Srdov war ein Hof, der an der Stelle der gegenwärtigen Siedlung Slaník liegt, vier Kilometer von Strakonitz Otava stromabwärts.

25 NA Praha ŘM, Urkunden, Nr. 2437, 2449.

26 Dazu vgl. schon L. Jan, Böhmische und mährische Adelige als Förderer und Mitglieder der geistlichen Ritterorden, in: The Crusades and the Military Orders. Expanding the Frontiers of Medieval Latin Christianity, ed. Z. Hunyadi, J. Laszlovsky, Budapest 2001, S. 303-317, hier vor allem 307-308. 
welchen Beweggründen sie dem Orden beigetreten sind. Diese stellten aber nicht eine definierbare Gruppe dar.

Die Ordensgeistlichen entstammten dagegen eher dem städtischen Milieu, und zwar sowohl dem deutschen, als auch dem böhmischen. Es wäre zweifellos interessant die Situation in den Konventen, die gegenseitige Beziehungen dieser zwei Gruppen oder die Präferenz einer der Verständigungssprachen zu untersuchen, dafür sind aber die Quellen zu wenig aussagekräftig.

\section{SUMMARY \\ Selected careers of Hospitallers in Bohemia in the $14^{\text {th }}$ century}

The article analyses the careers and backgrounds of Hospitallers serving in the Czech Priory of the Order. The study discusses the limited character of sources regarding Hospitallers in Bohemia in the $13^{\text {th }}$ century and presents in this context the careers of three members of the Order: Czech prior John of Zweretitz (Zvíretice), Prague Commander Pesslinus, who probably originated from a burgher family and John of Kladrubce who was a member of an insignificant aristocratic family near Strakonice. Secondly, the author demonstrates that members of the Czech Priory of the Order of St. John in the $14^{\text {th }}$ century came mainly from three major groups including members of the powerful Markwartinger family from Northern Bohemia, members of burgher families from Prague and members of the lesser noble family from around Strakonice, who were dependent on the lords Bawor of Strakonice or on the local convent of St John. 
